\title{
The PREvention Program for Alzheimer's RElated Delirium (PREPARED) cluster randomized trial: a study protocol
}

\author{
Machelle Wilchesky ${ }^{1,2,3,4^{*}}$ (D), Stephanie A. Ballard ${ }^{1,2}$, Philippe Voyer ${ }^{5,6}$, Jane McCusker ${ }^{7,8}$, Ovidiu Lungu 1,9,10, \\ Nathalie Champoux ${ }^{11}$, T. T. Minh Vu ${ }^{12}$, Martin G. Cole ${ }^{13}$, Johanne Monette ${ }^{3}$, Antonio Ciampi ${ }^{7,8}$, Eric Belzile ${ }^{8}$, \\ Pierre-Hugues Carmichael ${ }^{6}$ and Ted McConnell ${ }^{14}$
}

\begin{abstract}
Background: Delirium is a significant cause of morbidity and mortality among older people admitted to both acute and long-term care facilities (LTCFs). Multicomponent interventions have been shown to reduce delirium incidence in the acute care setting (30-73\%) by acting on modifiable risk factors. Little work, however, has focused on using this approach to reduce delirium incidence in LTCFs.

Methods: The objective is to assess the effectiveness of the multicomponent PREPARED Trial intervention in reducing the following primary outcomes: incidence, severity, duration, and frequency of delirium episodes in cognitively impaired residents. This 4-year, parallel-design, cluster randomized study will involve nursing staff and residents in 45-50 LTCFs in Montreal, Canada. Participating public and private LTCFs (clusters) that provide 24-h nursing care will be assigned to either the PREPARED Trial intervention or the control (usual care) arm of the study using a covariate constrained randomization procedure. Approximately 400-600 LTC residents aged 65 and older with dementia and/ or cognitive impairment will be enrolled in the study and followed for 18 weeks. Residents must be at risk of delirium, delirium-free at baseline and have resided at the facility for at least 2 weeks. Residents who are unable to communicate verbally, have a history of specific psychiatric conditions, or are receiving end-of-life care will be excluded. The PREPARED Trial intervention consists of four main components: a decision tree, an instruction manual, a training package, and a toolkit. Primary study outcomes will be assessed weekly. Functional autonomy and cognitive levels will be assessed at the beginning and end of follow-up, while information pertaining to modifiable delirium risk factors, medical consultations, and facility transfers will be collected retrospectively for the duration of the follow-up period. Primary outcomes will be reported at the level of intervention assignment. All researchers analyzing the data will be blinded to group allocation.
\end{abstract}

Discussion: This large-scale intervention study will contribute significantly to the development of evidence-based clinical guidelines for delirium prevention in this frail elderly population, as it will be the first to evaluate the efficacy of a multicomponent delirium prevention program translated into LTC clinical practice on a large scale.

Trial registration: NCT03718156, ClinicalTrials.gov.

\footnotetext{
*Correspondence: machelle.wilchesky@mcgill.ca

${ }^{1}$ Centre for Research in Aging, Donald Berman Maimonides Geriatric

Centre, 5795 Ave Caldwell, Montreal, QC H4W 1W3, Canada

Full list of author information is available at the end of the article
} original author(s) and the source, provide a link to the Creative Commons licence, and indicate if changes were made. The images or other third party material in this article are included in the article's Creative Commons licence, unless indicated otherwise in a credit line to the material. If material is not included in the article's Creative Commons licence and your intended use is not permitted by statutory regulation or exceeds the permitted use, you will need to obtain permission directly from the copyright holder. To view a copy of this licence, visit http://creativecommons.org/licenses/by/4.0/. The Creative Commons Public Domain Dedication waiver (http://creativeco mmons.org/publicdomain/zero/1.0/) applies to the data made available in this article, unless otherwise stated in a credit line to the data. 
Keywords: Delirium, Delirium superimposed on dementia, Modifiable risk factors, Multicomponent intervention, Nursing, Long-term care

\section{Background}

Delirium is a severe neuropsychiatric syndrome with acute onset and a fluctuating course that is characterized by disturbances in cognition, consciousness and attention [1]. It is a significant cause of morbidity and mortality, and is highly prevalent among older individuals across healthcare settings (14-56\% reported in acute care [2], $58-75.6 \%$ in intensive care units [3], and $1.4-70.3 \%$ in long-term care facilities (LTCFs)) [4]. Dementia is an important risk factor for developing delirium among elderly patients [5-7]: the risk of developing delirium is six-times greater among older individuals with dementia when compared to those without the disease [5], and prevalence rates of delirium superimposed on dementia range from 22 to $89 \%$ among populations aged 65 and older [8]. This is of particular concern in LTCFs, where dementia is common among the resident population [9]. Residents in LTCFs are also at an increased risk of developing delirium as a function of their frailty, cognitive impairment, and multiple medical comorbidities [10,11]. Indeed, more than $40 \%$ of LTCF residents experience at least one delirium episode over the course of their stay [12], leading to an increased number of falls, accelerated functional decline, increased mortality, and higher healthcare costs [1]. Despite these consequences, delirium recognition is known to be poor. Up to two-thirds of delirium cases are missed by physicians and nurses due to its fluctuant and variable nature [13], and it has been reported that $87 \%$ of delirium episodes that are superimposed on dementia go undetected in LTCFs [14].

Delirium prevention is essential $[15,16]$, as the risk of failure to return to baseline among survivors is high, even after adjustment for factors such as age and premorbid function [17]. Previous studies have found that $30-40 \%$ of delirium cases are preventable with the use of delirium protocols [11], and one American study estimates potential healthcare savings of up to 16 billion dollars annually [18]. Several multicomponent delirium prevention programs (MDPP) have successfully reduced delirium incidence in the acute care setting (30-73\% reduction) [1, 18-22]. Independent of type of acutecare ward or unit and level of cognitive decline, a 2015 systematic review of randomized trials of multicomponent interventions reported a relative reduction of $27 \%$ (RR 0.73, 95\%CI: 0.63-0.85) in delirium incidence [1]. Similarly, a systematic review that analyzed non-randomized studies reported an overall reduction of $63 \%$ (RR 0.37, 95\% CI: 0.27-0.53) [1]. Such interventions have also been associated with an overall reduction in hospital stay duration and mortality rate [23]. Although the wide range of estimates make it difficult to ascertain the degree to which delirium incidence can be reduced, it is clear that these interventions do have an impact in the acute care setting. The extent to which these findings can be extrapolated to the LTC setting, however, is unknown. For example, the studies listed in the above-mentioned acute care systematic reviews included patients who are younger than LTC residents (average age of 79 in acute care versus 85 in LTC) [1, 18], and who have shorter lengths of stay (average 5-38 days in acute care versus 2.5 years for LTC) $[1,18]$. The prevalence of dementia is also different in acute care versus LTC (but this information is not available for the above-mentioned studies).

According to both a 2014 Cochrane Database Systematic Review and the National Institute for Health and Clinical Excellence, research to assess the effectiveness of an MDPP in LTC is urgently needed [24, 25]. Despite this call to action and the increased risk of developing delirium in the LTC population, no multicomponent intervention based on modifiable risk factors had been evaluated and translated into LTC clinical practice to date at the time that we conceived the present trial [19]. However, in 2020, Boockvar et al. published a trial measuring the efficacy of a different multicomponent intervention targeting delirium risk factors (specifically, cognitive impairment, immobility, dehydration, and malnutrition) in LTC, the Hospital Elder Life Program (HELP-LTC) [26]. The HELP-LTC targeted a limited sample size $(n=219)$, and the intervention did not demonstrate its intended effect: no significant differences were found in delirium or delirium severity between the intervention and usual care groups [26]. A multitude of factors are listed by the authors to explain this null finding, including baseline differences in cognitive function between groups, greater than expected improvements on delirium severity and cognition, and novel adaptations of the intervention [26].

In contrast, an integrated knowledge translation strategy [27] was used to develop the PREvention Program for Alzheimer's RElated Delirium (PREPARED Trial) intervention for use in a LTC setting. Key stakeholders who were familiar with the LTC context were identified early in the research planning process and consulted throughout all subsequent research phases. To address the fact that interventions that are effective in clinical trials may underperform in a real world setting [28], a participatory approach was then used to examine and 
test the feasibility and acceptability of the PREPARED Trial intervention in two LTC facilities in three 5-week implementation cycles [29]. A thorough evaluation of the effectiveness of the PREPARED Trial intervention in preventing delirium among at-risk LTC residents is now required.

\section{Study objectives}

The primary objective of this study is to compare the efficacy of the PREPARED Trial multicomponent intervention to that of usual care on delirium. Specifically, we aim to assess the effect of the PREPARED Trial multicomponent intervention on the incidence of delirium, severity of delirium episodes, duration of delirium episodes, and number of delirium episodes among at-risk, cognitively impaired residents in LTCFs. Our secondary objectives aim to: compare the efficacy of the PREPARED Trial intervention to that of usual care on the incidence of falls among cognitively impaired LTC residents; estimate the association between medication use and delirium incidence in LTCFs; estimate if there is an effect modification by motor subtype of delirium or by dementia subtype; and measure the prevalence of delirium in participating LTCFs. Finally, we will compare the effect of the multicomponent PREPARED Trial intervention on other health outcomes, including changes in: functional autonomy or social engagement, cognitive functioning, the number of transfers to acute care, the number of consultations with healthcare providers, and mortality rates. All objectives will be reported at the level of intervention assignment.

\section{Methods/design Study design}

The PREPARED Trial is a 4-year cluster randomized trial. The PREPARED Trial intervention will be implemented at the LTCF level. To reduce potential contamination between intervention and control study arms by nursing staff members who may provide replacement hours of employment at different units/floors within their LTCF, a cluster design was adopted such that participating LTCFs (clusters) will be assigned to either the PREPARED Trial intervention or the control (usual care) arm of the study. Nursing staff and LTC residents meeting trial inclusion criteria from all arms will be enrolled in the study. Residents will be followed for an 18-week period or until death or transfer to another facility.

A cluster randomized trial parallel design, employing a covariate constrained randomization (CCR) procedure [30,31], will be used to allocate LTCFs, rather than individual residents, to either the intervention or control study arm. Cluster randomized trials typically use clusters as the main unit of analysis, given that the intervention is conducted at the cluster level (in the current study, the LTCFs are the clusters). However, given that the number of clusters is usually much smaller than the total number of participants, a simple cluster randomization or a procedure based on stratification may yield study arms that are imbalanced and differ on key baseline cluster characteristics. A CCR approach will be used to address this issue, as it specifically aims to balance the distribution of important cluster characteristics or covariates across study arms [30-32]. In addition, given the need to assess each resident weekly for a followup period of 18 weeks and the logistical limitations (large geographical coverage, 45-50 LTCFs included, and limited work force) involved in such a trial, we will employ a sequential approach whereby sets of clusters (LTCFs) will be randomized into the two study arms in consecutive trial 'cycles' lasting 20 weeks each, including the twoweek screening period before enrolment (see Fig. 1 for a breakdown of research activities within a study cycle).

This 18-week period was selected using data from one of the precursor delirium studies conducted in this population, which found that $97 \%$ of all incident episodes of delirium among cognitively impaired residents occurred within the first 18-weeks of follow-up [33]. Research staff will visit each facility to enroll eligible residents and to conduct follow-up assessments. Data will also be abstracted from resident charts and existing LTCF databases.

\section{Participants}

Participants for this study will include LTC residents (who will be assessed for delirium, cognition, functional autonomy, and other clinical/demographic variables) and nursing staff members (who will provide data pertaining to the resident population and/or implement the intervention). No monetary incentives will be provided to remunerate participation. Participating LTCFs will include public and semi-private residential LTCFs in and around Montreal, Quebec that provide residence to elderly individuals requiring specialized care per day, including assistance with one or more basic activities of daily living beyond that which can be provided in a community setting [34, 35]. Institutions will only be eligible if they provide $24-\mathrm{h}$ of supervised nursing care (i.e. an $\mathrm{RN}$ is on site $24 \mathrm{~h}$ per day), including professional health services and personal care.

All Registered Nurses (RNs), Licensed Practical Nurses (LPNs) and personal support workers (PSWs) working during the day shift will be eligible to participate if they: 1 ) are working at least 3 days/week on the same nursing unit (i.e. are not 'floating' between units); and 2) have 


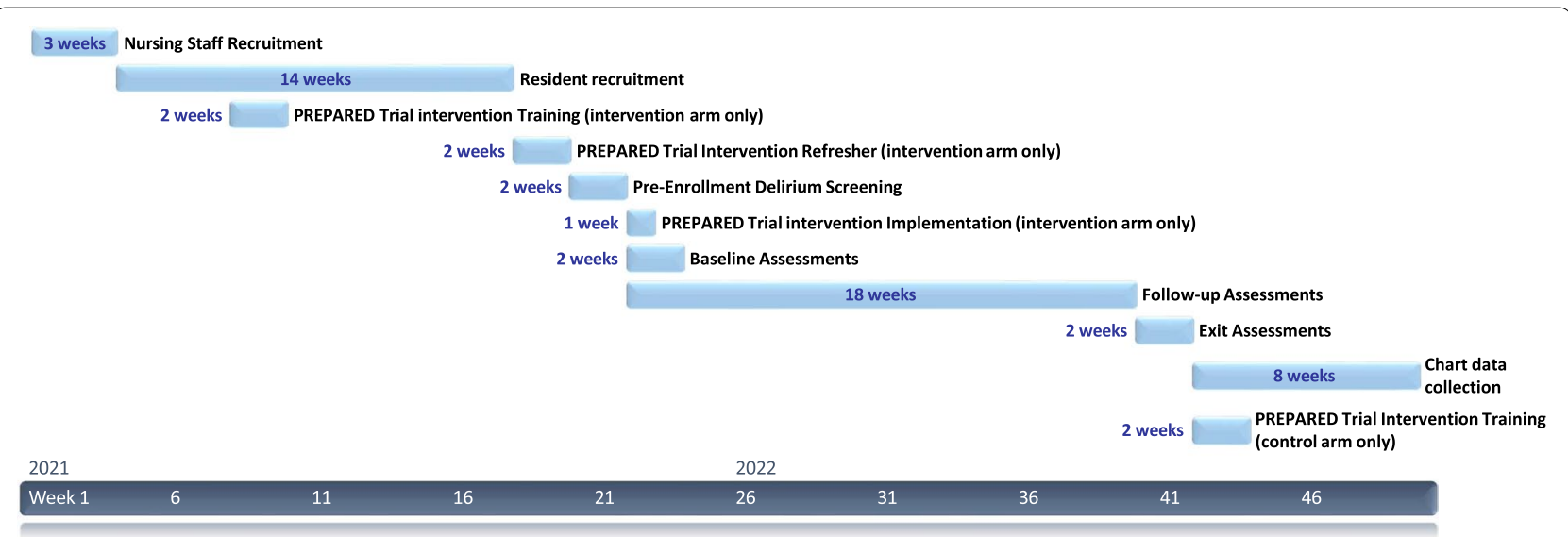

Fig. 1 Timeline of Research Activities per Trial LTC Facility

worked at the LTCF for at least 1 month prior to the follow-up start date at their site.

Residents will be enrolled based on a series of inclusion/exclusion eligibility criteria. In order to be included, residents must: be 65 years or older; have dementia and/ or cognitive impairment, as determined by discussions with the nursing staff and chart abstraction; have a minimum length of stay in the LTC facility of at least 2 weeks prior to the start of the baseline assessments; be at risk of delirium, as indicated by a score of 1 or higher on a validated 5-item delirium risk screening tool [36] (Fig. 2); and be delirium-free at baseline, as assessed by the Confusion Assessment Method (CAM) [37, 38], the Delirium Index (DI) [39], and a brief chart review over a period of two consecutive weeks immediately preceding the start of the follow-up period. Residents will be excluded if they are: unable to communicate verbally (as determined by either the nursing staff or two consecutive 0-score administrations of the composite cognitive interview, which is part of the CAM procedure); unable to communicate verbally in English or French; have a history of specific psychiatric conditions (bipolar disorder, depression with signs of psychosis, and psychotic disorders) or intellectual disability [40,41]; or are being provided with end-of-life or palliative care.

\section{Sample size calculation}

Based on sample size calculations, we plan to recruit between 400 and 600 LTC residents in the current study. Sample size was computed for our primary objective, to detect any differences between our intervention and control trial arms in the incidence of a first delirium episode after 18 weeks of follow-up. We used the Freedman log-rank test method to compare two survivor functions at the end of study follow-up. In a post-hoc secondary analysis of the precursor LTC delirium study, for which we applied the PREPARED Trial inclusion and exclusion criteria [33], we obtained incidence rates that we assume will be experienced by our control group, namely 3.75 cases per 100 person-weeks. We tested different scenarios concerning the size of our sample after 18 weeks of follow-up from baseline. We also tested our assumptions regarding the magnitude of our intervention effect by allowing the reduction in incidence rates to vary between 30 and 73\%, as previous studies (in non-LTC settings) have reported this range in variation [1, 18-22]. Using methods of estimation 'uncorrected' for cluster randomization, we found that including 180 residents per trial arm (after assuming a 15\% rate of attrition over our 18 week follow-up period to account for deaths, transfers and drop-outs) will enable us to detect a $40 \%$ reduction in the incidence of delirium $(\mathrm{HR}=0.6)$ with a power of $80 \%$ at the 5\% level (assuming 2-tailed test and equal group size).

The 'corrected' sample size for cluster randomization was then computed according to Rutterford et al. (2015) [42], where a design effect was incorporated that inflated the preliminary uncorrected calculation [43]. This design effect depends on two variables: 1) the Intra-cluster Correlation Coefficient (ICC) and 2) the number of clusters, where the total estimated required sample size is directly proportional to the magnitude of ICC and inversely proportional to the total number of clusters participating in the trial. Our sample size analysis, therefore, provides estimates using two conservative values for our expected outcome, and incorporates scenarios for both ICC and the number of clusters (or LTC facilities) that will be included in the study (Fig. 3).

For the purpose of this protocol, we began with an estimated ICC derived from the 2011 Quebec delirium 
The Delirium Prevention Program was developed and tested by a research team led by Jane McCusker MD, DrPH (Epidemiology, McGill University) and Philippe Voyer inf, PhD (Nursing, Université Laval).

Research was funded by the Alzheimer Society of Canada, the Canadian Nurses' Foundation and CIHR's Institute of Aging.

Formore information, contact jane.mccusker@mcgill.ca

\section{Decision tree:}

Program for the prevention of delirium in residents aged 65 and over who experience cognitive impairment*

\section{Is the resident at risk?}

$\square$ Can not name the province.

$\square$ Completely dependent for personal hygiene care.

$\square$ Constantly repeats a senseless action or motion.

$\square$ Fidgets, unable to sit still.

$\square$ Irritability (easily irritated or angered).
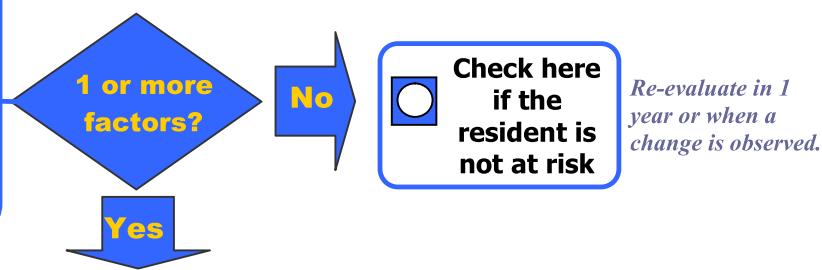

\section{Apply the optimal stimulation protocol}

\section{TNP \\ STIMUL}

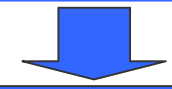

Evaluate the modifiable risk factors and plan corresponding interventions

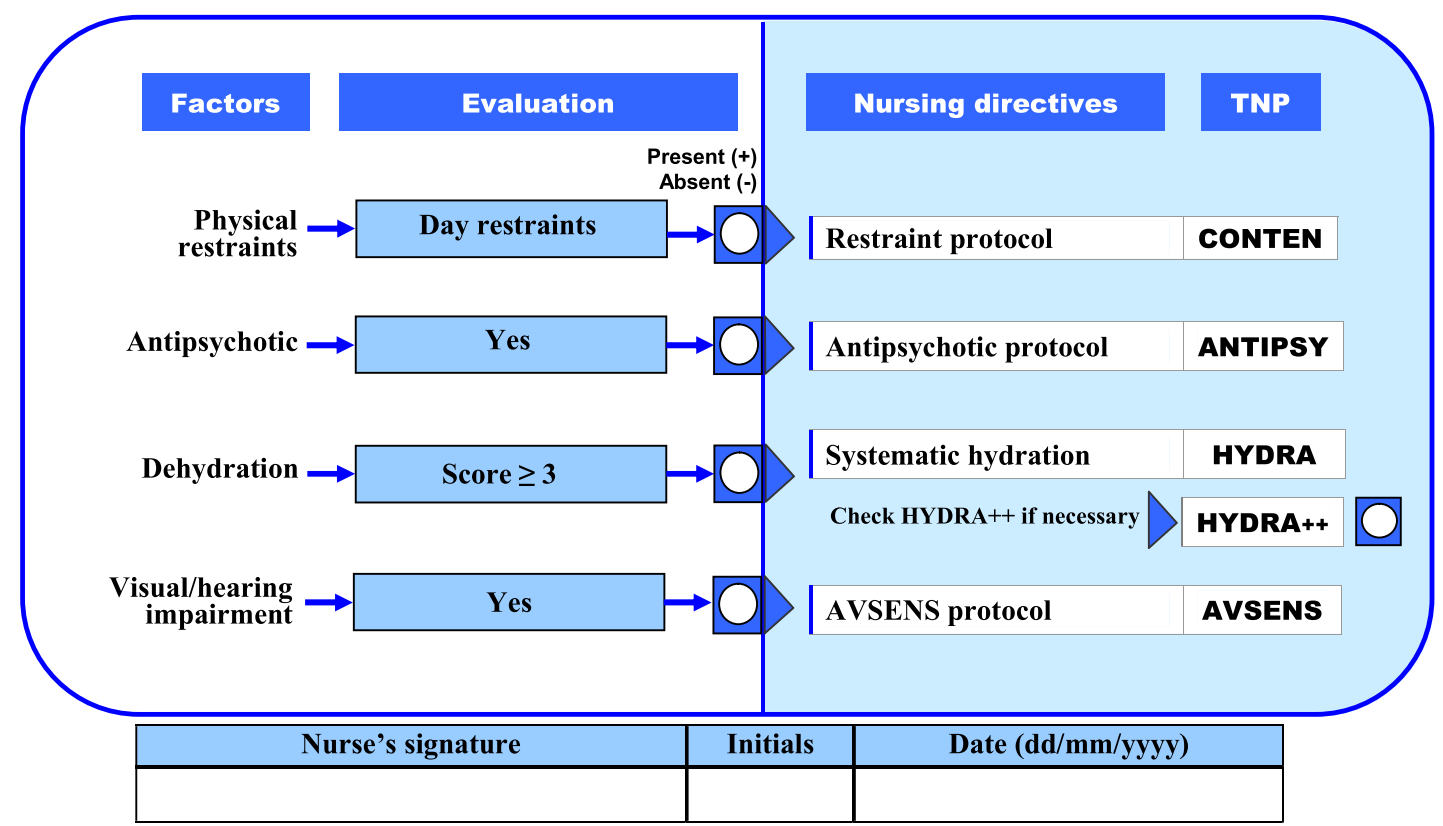

* This program is not intended for residents who have an intellectual disability or a history of psychiatric conditions listed in their chart (bipolar disorder, depression with signs of psychosis, and psychotic disorder).

Fig. 2 Delirium Risk Screening Tool and Decision Tree 


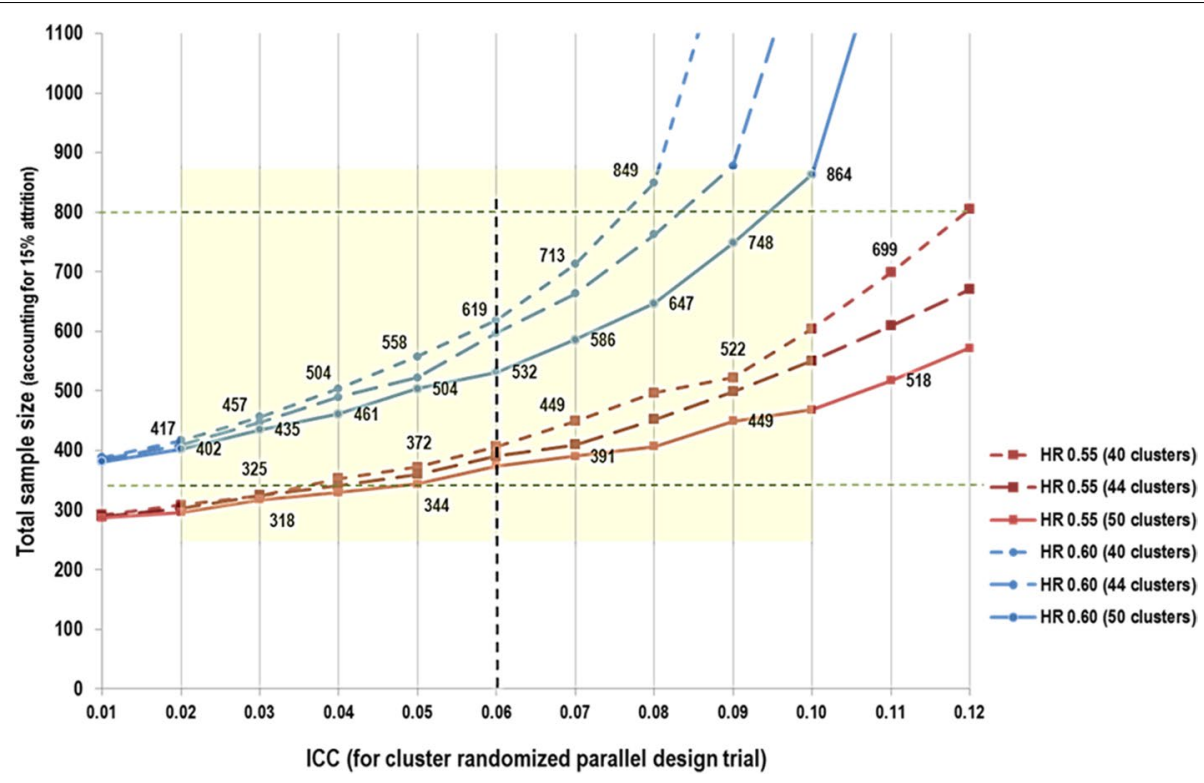

Fig. 3 PREPARED Cluster Randomized Trial Sample Size Estimation

study post-hoc analysis described above. In this dataset, when considering the three facilities that would be representative of the clusters or LTCFs participating in the PREPARED Trial, we found an ICC of 0.06. This estimate should be regarded as quite conservative, given that this ICC was computed using a total of only 3 clusters, whereas our study will be approximately 15 times larger in terms of total cluster size. The number of clusters per arm in our study will vary between 20 (worst case scenario) and 25 (best case scenario). Before performing our CCR procedure, recruited RNs will each be asked to provide the study team with the maximum number of participants that they would be willing to manage as trial participants under the assumption that theirs could be an intervention site. Given that we know a priori that our clusters are likely to be unequal in size, our randomization procedure will use this nurse-to-resident participant ratio as a CCR variable to balance the ratio of intervention-to-control participants in our trial. It should be noted that the range of sample size required varies greatly by ICC: from 864 study subjects within 50 clusters in order to detect a minimum HR of 0.6 in our worst case scenario of an ICC of 0.1 , to approximately 300 subjects for HR of -0.55 and an ICC of 0.02. As such, we plan to enroll about 200-300 residents per intervention arm, for a total enrollment of 400-600 residents, which will account for a liberal 15\% estimate for participant attrition. Computations for the above sample size calculations were performed using STATA version 13.0.

\section{Enrollment and randomization procedure}

To ensure a manageable workload for the LTCF nursing staff, the study will enroll only as many residents per team as the participating day shift $\mathrm{RN}$ deems to be reasonable. This number will be determined on an individual basis, and will constitute a contract between the research team and the LTCF RN.

Residents who have consented to participate will undergo a two-week delirium prevalence screening using the CAM and the DI (see assessments below) [37-39]. Residents who are delirium-free throughout this twoweek period will be randomized for inclusion in the study. The number randomized per unit will be consistent with the number agreed upon by that specific unit nurse.

Residents will be randomized using an electronic data management (EDM) platform provided by the Information Management Services Unit (IMS) at McGill University/Lady Davis Institute of the Jewish General Hospital. IMS will employ simple random sampling, a basic sampling technique where a subset of individuals (a sample) is selected from within a larger set of individuals (a population). In such a method, each individual is chosen entirely by chance, and each member of the population has an equal chance of being included in the sample.

\section{The PREPARED Trial intervention}

This PREPARED Trial intervention was developed by members of our team specifically for the LTC environment using an integrated knowledge translation strategy, which affirmed its feasibility in this setting [27]. 
The PREPARED Trial intervention is a multicomponent intervention (available in both English and French) consisting of the following:

1. Decision Tree (Fig. 2): This one-page support tool is a 4-step algorithm designed to guide nursing decisionmaking.

Step 1 consists of identifying residents at risk of developing delirium using the validated 5-item delirium risk screening tool [36]. For residents deemed at-risk, the tool will then guide the LTC nurse through the three following steps (below).

Step 2 describes the application of a protocol designed to provide optimal stimulation, and is intended to be implemented by all LTC nursing staff members to all intervention arm participants. The stimulation protocol (STIMUL) involves three actions: 1) surveying the use of eyeglasses and hearing aids, the room lighting, and organization of the space; 2) orienting the resident to time (season, month, day) and to space (city, room); and 3) stimulating the resident with the help of familiar objects, photos, and life histories.

Step 3 involves a set of specific nursing evaluations to assess the presence of the following five additional modifiable delirium risk factors (i.e., in addition to stimulation): 1) restraint use, 2) prolonged antipsychotic use, 3) dehydration, 4) vision problems, and 5) hearing problems) in preparation for the next step, which represents the specific PREPARED Trial intervention. Evidence of restraint and antipsychotic use are evaluated by examining the resident file, while dehydration is assessed via a thorough inspection of the skin and the mucous membrane of the mouth. Vision problems are evaluated using a tracking test, where residents are asked to follow the trajectory of a red ball within their field of vision. Hearing is assessed via a 'finger rubbing' test, where residents are asked to identify or react to sound, depending on their level of cognitive impairment.

Step 4 describes six specific interventions aimed at reducing the impact of specific risk factors found to be present in Step 3. These six specific interventions are the core of the PREPARED Trial intervention, and are designed to reduce the impact of antipsychotic use, sensory impairment (vision and hearing), restraint use, and dehydration. They are: i. Antipsychotic Intervention (ANTIPSY): A letter signed by the study clinician team recommending a re-evaluation of the resident's need for their antipsychotic medication as per clinical guidelines is inserted into the resident chart so that it may be read and signed by the treating physician.

ii. Vision Intervention (AVSENS-VISION): A note is made in the resident chart, consultations with an optometrist are suggested, and a relevant intervention based on clinical judgement is suggested and/ or implemented as needed.

iii. Hearing Intervention (AVSENS-AUDIT): A note is made in the resident chart, consultations with an audiologist are suggested, and a relevant intervention based on clinical judgement is suggested and/ or implemented as needed.

iv. Restraint Intervention (CONTEN): All restraints are removed during care that requires the constant presence of a caregiver (nail care, feeding, and dressing changes).

v. Hydration Intervention (HYDRA): A brightly colored container of fresh water is placed on the bedside table, the resident is offered liquids that $\mathrm{s} / \mathrm{he}$ enjoys and at least one glass of liquid at each meal, $60-180 \mathrm{ml}$ of liquid is offered during medication administration, and a glass of liquid is offered both after morning care and in the middle of the afternoon.

vi. Hydration Intervention Plus (HYDRA++): For residents who are hesitant to drink, $60 \mathrm{ml}$ of liquid is offered each time a caregiver enters the room in addition to the HYDRA protocol.

It is important to note that AVSENS-AUDIT represents a novel addition to the PREPARED Trial intervention. AVSENS, which stands for Auditory/Vision/Sensory, expands on the original VISION protocol as published by Voyer et al. (2014) to include both a visual and auditory evaluation/intervention [29]. This expansion was created to reflect the fact that hearing impairment (not just visual impairment) has been shown to be a risk factor for developing delirium in this population [21, 44, 45]. Furthermore, existing delirium prevention strategies have been shown to improve clinical outcomes by addressing hearing impairment [21].

In order to account for the possible effects of sensory impairment on cognitive testing [46-49], residents will be offered 'Pocketalkers' ${ }^{\text {TM }}$, or personal amplification devices, to use during trial assessments. Use of these auditory aids has been recommended as one of the strategies to avert the misdiagnosis or over-diagnosis 
of cognitive impairment in older adults with sensory impairment, and to improve the specificity of cognitive testing [50]. All personal amplification devices will be cleaned and sanitized with alcohol-based sanitation wipes prior to each use.

These six trial interventions will be integrated (where indicated) into the Therapeutic Nursing Plan (TNP) by the LTCF RNs who are mandated to carry out both the antipsychotic and the vision/hearing interventions. The restraint and hydration protocols, however, will be administered by LPNs and PSWs.

2. Instruction Manual: This 3-page, easy-to read, manual provides detailed instructions on how to evaluate the 4 specific delirium risk factors. Specific content of each intervention is explained, and nursing directives are provided.

3. Training Package: Provided by the research staff, the package includes (1): a tailored PowerPoint presentation to nurses $(60 \mathrm{~min})$ and LPNs/PSWs (30 min) covering all of the information needed to effectively use the PREPARED Trial intervention; and (2) delirium rounds on the unit during the follow up period (organized by the CRN, as needed) to discuss PREPARED Trial intervention-related issues. Training sessions for regulated staff members (i.e. RNs and LPNs) were accredited by Université Laval.

4. Toolkit: This transparent box contains all of the necessary materials to evaluate risk factors (e.g., a red ball for the visual assessment protocol) and implement the interventions (e.g. voice amplifiers, magnifiers), as well as memory aid tools (poster, lanyard memory card, leaflet and notice board listing targeted residents). A box will be provided to each unit in the intervention arm of the study.

The intervention components are based on current best practices in LTC, and are not resource intensive an important consideration in the context of budgetary constraints and reduced direct care time in this healthcare setting. This trial intervention has been shown to be both feasible and acceptable by Quebec LTC staff [29]. The intervention protocol is low-risk, non-invasive, and personalized to meet the risk profiles of each participant. Participants who no longer meet study inclusion criteria or who are no longer able to engage in the study and its protocols due to disease deterioration (as declared by the primary $\mathrm{RN}$ ) will stop receiving the intervention and all study evaluations. Trial participants are free to withdraw from the study at any time.

\section{Adherence to the protocol and nursing staff satisfaction assessment}

Adherence to the PREPARED Trial intervention (as documented by the addition of the intervention protocols to the TNP) in the intervention arm will be evaluated by the CRN. In addition, RAs will record the use of hearing/vision aids, and the presence of water and restraints (AVSENS-VISION, AVSENS-AUDIT, HYDRA, HYDRA++, and CONTEN) at every weekly face-to-face assessment with residents during the follow-up period. Nursing staff perceptions of the PREPARED Trial multicomponent intervention will be evaluated at study end using a questionnaire to assess intervention relevance, clarity, perceived burden, and sustainability [29].

\section{Blinding}

LTCFs will not be blinded to group allocation (i.e. control/intervention), as trial arm allocation will obvious (the nursing staff in the intervention group will be trained prior to their participation and receive toolkits and reminder posters, while those in the control group will not). Similarly, the RAs tasked with conducting resident assessments will also not be blinded to group allocation, as they will be constantly exposed to the everyday workings of participating LTCFs. For example, certain visual cues (i.e. materials in the charting room) may indicate the trial status of many LTCFs. However, all research staff members responsible for abstracting information pertaining to modifiable delirium risk factors, medical consultations, and any LTCF transfers will be blinded to resident assessment scores, and should engage in such tasks with impartiality. The research team member administering the CAM/DI for a given resident will also be blinded to the cognitive level assessment of that resident. Finally, all researchers tasked with analyzing the data obtained from this study will be blinded to group allocation, as all LTCF names and randomization statuses will be automatically anonymized prior to data analysis by IMS.

\section{Assessments}

As part of the eligibility procedure, delirium risk will be assessed using a validated five-item delirium risk screening tool (see "Is the resident at risk?" section of the Decision Tree, Fig. 2) [36]. To be included for study, a resident will require a score of 1 or higher on this scale. Prevalence of delirium will be then assessed weekly for 2 weeks at baseline (screening), and only residents with two consecutive negative CAM scores will be considered for trial inclusion. Delirium incidence and severity (primary outcomes) will be assessed weekly during the 18-week follow-up period. These delirium assessments will be completed using the CAM [37, 38] and the DI [39] 


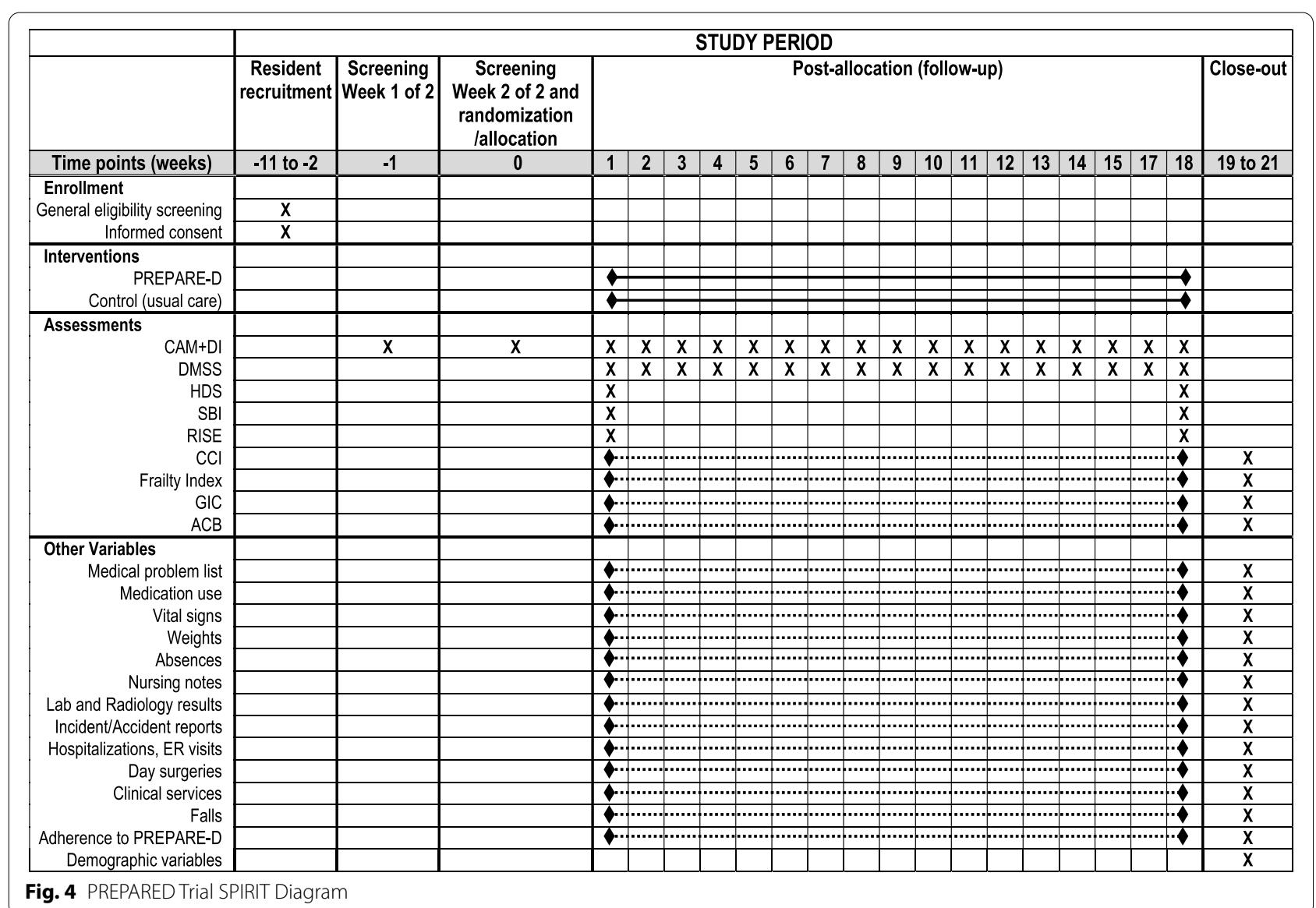

as part of a standardized interaction with the resident based on a series of questions adapted using items from various cognitive assessment tools, specifically the MiniCOG (memory) [51-53], Montreal Cognitive Assessment (orientation) and Hierarchical Dementia Scale (concentration) [36, 40, 51, 54, 55]. Functional autonomy will be evaluated at the beginning and end of the followup period (weeks 1 and 18) using the Shah version of the Barthel Index [56], an ordinal scale used to measure independence in the activities of daily living (ADLs) adapted to the LTCF environment. Cognitive function will be also be evaluated at these time points using the Hierarchic Dementia Scale (HDS) [55]. Resident comorbidity will be assessed via chart review and reported using the Charlson Comorbidity Index [57] and Geriatric Comorbidity Index [58]. Resident frailty will be assessed via a combination of comorbidity and other measures of wellbeing, such as functional autonomy, social engagement, and healthcare utilization [59]. Medication information and anticholinergic drug use will also be extracted from resident medical charts, and assessed using the Anticholinergic Burden Scale [60]. Fig. 4 presents a visual representation of the enrolment, intervention, and assessment schedule (SPIRIT Diagram).

\section{Statistical data analysis}

All statistical analyses will be conducted using SAS version 9.4, STATA version 13.0, and R software. Our study has four primary objectives, four secondary, and six tertiary objectives, all aimed at comparing the effect of our PREPARED Trial intervention to that of usual care. Data analysis will be conducted and reported in accordance with current CONSORT guidelines [61, 62].

For each outcome, we will report the estimates of the regression coefficients obtained from the model for the intervention: Hazard Ratio for survival analysis, beta estimate for the continuous outcome, Incident Rate Ratio for the count outcome, and Odds Ratio for the binary outcome. We will also report the ICC (ratio of the variance explained by the LTCF to the total variance explained by the model). All analyses will be conducted at alpha 0.05 and $95 \%$ confidence intervals will be reported.

Missing or incomplete outcome data will inevitably appear in our study. Our general approach will be based 
on intention-to-treat, using inverse probability weighting to account for missing data [63, 64]. To assess the appropriateness of this approach to missing data, we will conduct two sensitivity analyses using the following approaches: 1) inclusion of subjects with complete data only; and 2) use of multiple imputation for missing data $[65,66]$.

\section{Quality assurance}

In order to ensure that assessments are standardized across evaluators, RAs and CRNs were trained and supervised in Quebec City by a world-renown geriatric nurse with prior experience administering the PREPARED Trial intervention (P. Voyer, PhD). These training sessions included bedside practice. In addition, to mitigate the risk of missing data due to illness or personnel attrition (which is a real possibility given the continuous and inflexible nature of the assessment schedule), several additional researchers will also be trained in study procedures as replacement evaluators, and the study team will be coached in how to train new employees (i.e. 'train-thetrainer'). Finally, the research staff will also be provided with "booster sessions" periodically, in order to ensure consistency in the administration of the assessments.

The CRN was trained and coached by the geriatric nurse. In turn, the CRN will offer coaching sessions throughout the study to all participating nurses. This will help to ensure standardized implementation of the PREPARED Trial intervention across nursing units.

We will also conduct inter-rater reliability testing during the two-week baseline assessments (prevalence screening), in order to ensure standardized training and assessments [33]. During this period, each RA will accompany a fellow RA on three delirium assessments, so as to independently observe and score the same resident under the same conditions. RAs will also conduct the weekly follow-up assessments in rotating shifts (i.e. will spend no more than three consecutive weeks with the same participants), so as to ensure that residents are assessed by multiple RAs and that assessment results are not nested within an individual assessor.

\section{Data monitoring and management}

The logistical demands associated with large randomized trials involving intensive assessment schedules can be overwhelming. As such, we have contracted with the IMS Unit at McGill University/ the Lady Davis Institute of the Jewish General Hospital to provide an EDM platform that is accessible through a secured online portal. Research staff will enter all assessment scores, including all baseline, weekly, and end of follow-up scores, directly into personal computers (in tablet form) at the point of data collection. The same holds true for all data abstracted from resident charts. All tablets will have access to the Internet, and data collection modules will include scheduling functions for clinical assessments based on the week of follow-up for each study resident. The PREPARED Trial Coordinator and the Principal Investigator will oversee all management of the EDM platform, in collaboration with the IMS team. Basic information (i.e. name, floor, and contact information) pertaining to residents and nursing staff will be entered into the EDM platform. The Clinical Trial Coordinator will also monitor participant retention and follow-up on a weekly basis using the EDM platform. All information collected prior to participant withdrawal will be kept, analyzed or used to ensure the integrity of the project.

All sensitive personal information pertaining to residents and staff members that is not needed for analysis (but that will facilitate data collection, such as name and resident date of birth) will be stored separately and encrypted in a non-searchable table to preserve confidentiality. As a result of this feature, all data that are extracted from the database by the researchers will be automatically anonymized prior to data analysis. Study data will be stored on a secured online portal by IMS, until such time as it is extracted and saved by the Principal Investigator (who will manage the final trial dataset). Given that our trial intervention involves recognized good clinical practices, establishing a Data Safety and Monitoring Board was deemed to be unwarranted.

\section{Discussion}

This study will both advance health-related knowledge and significantly improve health outcomes via the demonstration of the effectiveness of the PREPARED Trial intervention in preventing delirium incidence, severity, frequency and duration of delirium episodes among atrisk LTC residents. New evidence generated by this trial will contribute significantly to the development of clinical guidelines for delirium prevention in this frail elderly population, and will provide a blueprint of a program that can be transferred to other LTCFs around the world. Due to the high prevalence of delirium in this population and its serious consequences on morbidity and mortality, this thorough and well-designed large-scale intervention has the potential to reduce healthcare costs [67] and to significantly improve the quality of LTC in Canada and beyond.

\section{Abbreviations}

ACB: Anticholinergic Burden Scale; ANTIPSY: Antipsychotic intervention component of the PREPARED Trial; AVSENS-AUDIT: Hearing intervention component of the PREPARED Trial; AVSENS-VISION: Vision intervention component of the PREPARED Trial; CAM: Confusion Assessment Method; CCI: 
Charlson Comorbidity Index; CCR: Covariate constrained randomization; CONTEN: Restraint intervention component of the PREPARED Trial; CRN: Clinical research nurse; DI: Delirium Index; DMSS: Delirium Motor Subtype Scale; EDM: Electronic data management; GIC: Geriatric Index of Comorbidity; HDS: Hierarchic Dementia Scale; HELP: Hospital Elder Life Program; HYDRA: Hydration intervention component of the PREPARED Trial; HYDRA++: Hydration plus intervention component of the PREPARED Trial; ICC: Intra-cluster Correlation Coefficient; IMS: Information Management Services, the unit at McGill University/ the Lady Davis Institute of the Jewish General Hospital that will provide the electronic data management platform; LPN: Licensed Practical Nurse; LTC: Long-term care (the study setting); LTCF: Long-term care facility; MDPP: Multicomponent delirium prevention program; PREPARED: PREvention Program for Alzheimer's RElated Delirium; PSW: Personal Support Worker; RA: Research Assistant; RISE: Resident Index of Social Engagement; RN: Registered Nurse (or LTCF nurse at a long-term care site); SBI: Shah version of the Barthel Index; STIMUL: Stimulation component of the PREPARED Trial intervention; TCPS: TriCouncil Policy Statement; TNP: Therapeutic Nursing Plan.

\section{Acknowledgements}

Not Applicable.

\section{Authors' contributions}

$M W, S A B$ and $O L$ wrote this protocol in consultation with PV, JMC, NC, TTMV, MGC, JM, AC, EB, PHC, and TM, who reviewed it and refined it based on the original proposal. All authors read and approved the final version of the manuscript.

\section{Funding}

The project was scientifically evaluated and approved by the Canadian Institutes of Health Research. Funding for this project was awarded to MW (PI), NC, AC, OL, JMC, JM, PV, and TTMV (Co-PIs) by the Canadian Institutes of Health Research (PJT-148937), and to MW (PI) by the Donald Berman Maimonides Medical Research Foundation.

These institutions will play no part in study design, collection, management, analysis, and the interpretation of data. There is an expectation, however, that we publish our trial results in open access journals.

\section{Availability of data and materials}

A de-identified dataset may be made available upon reasonable request of the corresponding author once the study is complete.

\section{Declarations}

\section{Ethics approval and consent to participate}

The Comité central d'éthique de la recherche du ministre de la Santé et des Services Sociaux du Québec provided ethical oversight and approved the study (ethics certificate number: CCER 17-18-04). The study will involve the recruitment of nursing staff and LTC residents. Nursing staff will be approached at a quiet moment during their work shift and will be asked to sign an informed consent after they have agreed to participate following the presentation of the study objectives and procedures.

Resident consent procedures will depend on resident capacity to consent, and will follow a protocol implemented by members of our research team in one of the precursor delirium studies conducted in this LTC population with dementia [33]. As in that study, competence to consent will be based on legal mandates or, in cases where no mandate is available, the clinical impressions of the primary nurse. As per Article 21 of the Civil Code of Québec and Article 3.9 of the TriCouncil Policy Statement (TCPS) (2014), authorized third party individuals (i.e. legal guardians or responsible family members) will be asked to provide consent on behalf of residents who are unable to provide informed consent themselves. In cases where the primary nurse has indicated incapacity to consent in the absence of legal documentation and a participant acquires or regains decision-making capacity during the course of the trial (as perceived by the primary nurse and reported to the research staff), the research staff will promptly seek the consent of the individual as a condition of continuing participation as per Article 3.8 of the TCPS (2014). In order to protect the confidentiality of LTC residents and their families, the research team will not have access to resident or proxy contact information initially. Instead, a list of potential study subjects will be identified based on general eligibility screening criteria by the RN or LPN responsible for direct care. The RN or LPN will then approach all residents who are eligible to participate (or their proxies) to briefly discuss the study and obtain permission to speak with the research team about their potential participation (either in person or by post). Only residents (or proxies) who have agreed to speak with the research team will then be asked to provide consent. All participant consent will be sought prior to group allocation (the CCR procedure) and resident randomization.

All important protocol modifications will be communicated to the Comité central d'éthique de la recherche du ministre de la Santé et des Services Sociaux du Québec as per their published guidelines and procedures.

\section{Consent for publication}

Not applicable.

\section{Competing interests}

The authors declare that they have no competing interests.

\section{Author details}

${ }^{1}$ Centre for Research in Aging, Donald Berman Maimonides Geriatric Centre, 5795 Ave Caldwell, Montreal, QC H4W 1W3, Canada. ${ }^{2}$ Department of Family Medicine, McGill University, 5858 Côte-des-Neiges Road, Montreal, QC H3S 1Z1, Canada. ${ }^{3}$ Division of Geriatric Medicine, McGill University, Jewish General Hospital, 3755 Cote St. Catherine Road, Room E-0012, Montreal, QC H3T 1E2, Canada. ${ }^{4}$ Centre for Clinical Epidemiology, Lady Davis Institute, Jewish General Hospital, 3755 Cote St. Catherine Road, Montreal, QC H3T 1E2, Canada. ${ }^{5}$ Faculty of Nursing Sciences, Laval University, Pavillon Ferdinand-Vandry, 1050 avenue de la Médecine, local 3645, Québec, QC G1V 0A6, Canada. ${ }^{6}$ Centre d'excellence sur le vieillissement de Québec, Centre intégré universitaire de santé et de services sociaux de la Capitale-Nationale, 1050 chemin Sainte-Foy, L2-30, Quebec City, QC G1S 4L8, Canada. ${ }^{7}$ Department of Epidemiology, Biostatistics and Occupational Health, McGill University, 1020 Pine Ave W, Montreal, QC H3A 1A2, Canada. ${ }^{8}$ St. Mary's Research Centre, 3830 Avenue Lacombe, Hayes Pavilion, suite 4720, Montreal, QC H3T 1M5, Canada. ${ }^{9}$ Functional Neuroimaging Unit, Centre de recherche de I'Institut universitaire de gériatrie de Montréal, 4565 Queen Mary Rd, Montreal, QC H3W 1W5, Canada. ${ }^{10}$ Department of Psychiatry, Université de Montréal, Pavillon Roger-Gaudry, Faculté de Medicine, C.P. 6128, succursale Centre-ville, Montreal, QC H3C 3J7, Canada. ${ }^{11}$ Faculty of Medicine, Université de Montréal, 2900 Boulevard Edouard-Montpetit, Montreal, QC H3T 1 J4, Canada. ${ }^{12}$ Centre de recherche du CHUM, 91000, rue Saint-Denis, Montréal, QC H2X 0A9, Canada. ${ }^{13}$ Department of Psychiatry, McGill University, Ludmer Research \& Training Building, 1033 Pine Avenue West, Montreal, QC H3A 1A, Canada. ${ }^{14}$ Division of General Internal Medicine, McGill University, 1001 Decarie Boulevard, Montreal, QC H4A 3J1, Canada.

Received: 28 July 2021 Accepted: 15 October 2021

Published online: 16 November 2021

\section{References}

1. Martinez F, Martinez F, Tobar C, Tobar C, Hill N. Preventing delirium: should non-pharmacological, multicomponent interventions be used? A systematic review and meta-analysis of the literature. Age Ageing. 2015;44(2):196-204

2. Fong TG, Tulebaev SR, Inouye SK. Delirium in elderly adults: diagnosis, prevention and treatment. Nat Rev Neurol. 2009;5(4):210.

3. Lin W-L, Chen Y-F, Wang J. Factors associated with the development of delirium in elderly patients in intensive care units. J Nurs Res. 2015;23(4):322-9.

4. de Lange $E$, Verhaak $P$, van der Meer K. Prevalence, presentation and prognosis of delirium in older people in the population, at home and in long term care: a review. Int J Geriatr Psychiatry. 2013;28(2):127-34.

5. Pisani MA, Murphy TE, Van Ness PH, Araujo KL, Inouye SK. Characteristics associated with delirium in older patients in a medical intensive care unit. Arch Intern Med. 2007;167(15):13-27.

6. McCusker J, Cole M, Abrahamowicz M, Han L, Podoba JE, RammanHaddad L. Environmental risk factors for delirium in hospitalized older people. J Am Geriatr Soc. 2001;49(10):1327-34. 
7. Pompei P, Foreman M, Rudberg MA, Inouye SK, Braund V, Cassel CK. Delirium in hospitalized older persons: outcomes and predictors. J Am Geriatr Soc. 1994;42(8):809-15.

8. Fick DM, Agostini JV, Inouye SK. Delirium superimposed on dementia: a systematic review. J Am Geriatr Soc. 2002;50(10):1723-32.

9. Voyer P, Richard S, Doucet L, Carmichael P-H. Predisposing factors associated with delirium among demented long-term care residents. Clin Nurs Res. 2009;18(2):153-71.

10. Kolanowski A, Mogle J, Fick DM, Hill N, Mulhall P, Nadler J, et al. Pain, delirium, and physical function in skilled nursing home patients with dementia. J Am Med Dir Assoc. 2015;16(1):37-40.

11. Voyer P, McCusker J, Cole MG, Khomenko L. Influence of prior cognitive impairment on the severity of delirium symptoms among older patients. J Neurosci Nurs. 2006;38(2):90.

12. Cheung ENM, Benjamin S, Heckman G, Ho JM-W, Lee L, Sinha SK, et al. Clinical characteristics associated with the onset of delirium among long-term nursing home residents. BMC Geriatr. 2018;18(1):1-7.

13. Thomas $E$. The effectiveness of non-pharmacological multi-component interventions for the prevention of delirium in non-intensive care unit older adult hospitalized patients: a systematic review. JBI Database Syst Rev Implementation Rep. 2014;12(4):180-232.

14. Voyer P, Richard S, Doucet L, Danjou C, Carmichael P-H. Detection of delirium by nurses among long-term care residents with dementia. BMC Nurs. 2008;7(1):1-14.

15. Cole MG. Persistent delirium in older hospital patients. Curr Opin Psychiatry. 2010;23(3):250-4.

16. Cole MG, McCusker J. Treatment of delirium in older medical inpatients: a challenge for geriatric specialists. J Am Geriatr Soc. 2002;50(12):2101-3.

17. Andrew MK, Freter SH, Rockwood K. Incomplete functional recovery after delirium in elderly people: a prospective cohort study. BMC Geriatr. 2005;5.

18. Hshieh TT, Hshieh TT, Puelle M, Dowal S, Travison T, Inouye SK, et al. Effectiveness of multicomponent nonpharmacological delirium interventions: a meta-analysis. JAMA Intern Med. 2015;175(4):512-20.

19. Reston JT, Schoelles KM. In-facility delirium prevention programs as a patient safety strategy: a systematic review. Ann Intern Med. 2013;158(5):375-80.

20. Vidán MT, Sánchez E, Alonso M, Montero B, Ortiz J, Serra JA. An intervention integrated into daily clinical practice reduces the incidence of delirium during hospitalization in elderly patients. J Am Geriatr Soc. 2009:57(11):2029-36.

21. Inouye SK, Bogardus ST Jr, Charpentier PA, Leo-Summers L, Acampora D, Holford TR, et al. A multicomponent intervention to prevent delirium in hospitalized older patients. N Engl J Med. 1999;340(9):669-76.

22. Gentric A, Le Deun P, Estivin S. Prévention du syndrome confusionnel dans un service de médecine interne gériatrique. Rev Med Intern. 2007;28(9):589-93.

23. Lundström M, Edlund A, Karlsson S, Brännström B, Bucht G, Gustafson Y A multifactorial intervention program reduces the duration of delirium, length of hospitalization, and mortality in delirious patients. J Am Geriatr Soc. 2005;53(4):622-8.

24. Clegg A, Siddiqi N, Heaven A, Young J, Holt R. Interventions for preventing delirium in older people in institutional long-term care. Cochrane Database Syst Rev. 2014;1:CD009537.

25. O'Mahony R, Murthy L, Akunne A, Young J, Guideline DG. Synopsis of the National Institute for health and clinical excellence guideline for prevention of delirium. Ann Intern Med. 2011;154(11):746-51.

26. Boockvar KS, Judon KM, Eimicke JP, Teresi JA, Inouye SK. Hospital elder life program in long-term care (HELP-LTC): a cluster randomized controlled trial. J Am Geriatr Soc. 2020;68(10):2329-35.

27. Lapaige V. "integrated knowledge translation" for globally oriented public health practitioners and scientists: framing together a sustainable transfrontier knowledge translation vision. J Multidiscip Healthc. 2010;3:33-47.

28. Bradley EH, Schlesinger M, Webster TR, Baker D, Inouye SK. Translating research into clinical practice: making change happen. J Am Geriatr Soc. 2004;52(11):1875-82.

29. Voyer P, McCusker J, Cole MG, Monette J, Champoux N, Vu M, et al. Feasibility and acceptability of a delirium prevention program for cognitively impaired long term care residents: A participatory approach. J Am Med Dir Assoc. 2014;15(1):77 e1-. e9.

30. Dickinson LM, Beaty B, Fox C, Pace W, Dickinson WP, Emsermann C, et al. Pragmatic cluster randomized trials using covariate constrained randomization: a method for practice-based research networks (PBRNs). J Am Board Fam Med. 2015;28(5):663-72.

31. Moulton LH. Covariate-based constrained randomization of group-randomized trials. Clin Trials. 2004;1 (3):297-305.

32. Nietert PJ, Jenkins RG, Nemeth LS, Ornstein SM. An application of a modified constrained randomization process to a practice-based cluster randomized trial to improve colorectal cancer screening. Contemp Clin Trials. 2009;30(2):129-32.

33. McCusker J, Cole MG, Voyer P, Monette J, Champoux N, Ciampi A, et al. Prevalence and incidence of delirium in long-term care. GPS Int J Geriatr Psychiatry. 2011;26(11):1152-61.

34. Berta W, Laporte A, Zarnett D, Valdmanis V, Anderson G. A panCanadian perspective on institutional long-term care. Health Policy. 2006;79(2-3):175-94.

35. Branch LG, Jette AM. A prospective study of long-term care institutionalization among the aged. Am J Public Health. 1982;72(12):1373-9.

36. McCusker J, Cole MG, Voyer P, Ciampi A, Monette J, Champoux N, et al. Development of a delirium risk screening tool for long-term care facilities. GPS Int J Geriatr Psychiatry. 2012;27(10):999-1007.

37. Inouye SK, van Dyck CH, Alessi CA, Balkin S, Siegal AP, Horwitz RI. Clarifying confusion: the confusion assessment method. A new method for detection of delirium. Ann Intern Med. 1990;113(12):941-8.

38. Wei LA, Fearing MA, Sternberg EJ, Inouye SK. The confusion assessment method: a systematic review of current usage. J Am Geriatr Soc. 2008;56(5):823-30.

39. McCusker J, Cole MG, Dendukuri N, Belzile E. The delirium index, a measure of the severity of delirium: new findings on reliability, validity, and responsiveness. JGS J Am Geriatr Soc. 2004;52(10):1744-9.

40. Voyer P, Richard S, Doucet L, Carmichael PH. Detecting delirium and subsyndromal delirium using different diagnostic criteria among demented long-term care residents. J Am Med Dir Assoc. 2009:10(3):181-8.

41. Voyer P, Champoux N, Desrosiers J, Landreville P, McCusker J, Monette $J$, et al. Recognizing acute delirium as part of your routine [RADAR]: a validation study. BMC Nurs. 2015;14(1):19.

42. Rutterford C, Copas A, Eldridge S. Methods for sample size determination in cluster randomized trials. Int J Epidemiol. 2015:44(3):1051-67.

43. Campbell MK, Fayers PM, Grimshaw JM. Determinants of the intracluster correlation coefficient in cluster randomized trials: the case of implementation research. Clin Trials. 2005;2(2):99-107.

44. Elie M, Cole MG, Primeau FJ, Bellavance F. Delirium risk factors in elderly hospitalized patients. J Gen Intern Med. 1998;13(3):204-12.

45. Galanakis P, Bickel H, Gradinger R, Von Gumppenberg S, Förstl H. Acute confusional state in the elderly following hip surgery: incidence, risk factors and complications. Int J Geriatr Psychiatry. 2001;16(4):349-55.

46. Schneider BA, Pichora-Fuller K, Daneman M. Effects of senescent changes in audition and cognition on spoken language comprehension. In The aging auditory system. New York: Springer; 2010. p. 167-210.

47. McCoy SL, Tun PA, Cox LC, Colangelo M, Stewart RA, Wingfield A. Hearing loss and perceptual effort: downstream effects on older adults' memory for speech. Q J Exp Psychol Sect A. 2005;58(1):22-33.

48. Rabbitt PM. Channel-capacity, intelligibility and immediate memory. Q J Exp Psychol. 1968;20(3):241-8

49. Rabbitt P. Mild hearing loss can cause apparent memory failures which increase with age and reduce with IQ. Acta Otolaryngol. 1991;111(sup476):167-76.

50. Dupuis K, Pichora-Fuller MK, Chasteen AL, Marchuk V, Singh G, Smith SL. Effects of hearing and vision impairments on the Montreal cognitive assessment. Aging Neuropsychol Cognit. 2015;22(4):413-37.

51. Borson S, Scanlan JM, Chen P, Ganguli M. The Mini-cog as a screen for dementia: validation in a population-based sample. J Am Geriatr Soc. 2003;51(10):1451-4

52. Borson S, Scanlan J, Brush M, Vitaliano P, Dokmak A. The mini-cog: a cognitive 'vital signs' measure for dementia screening in multi-lingual elderly. Int J Geriatr Psychiatry. 2000;15(11):1021-7.

53. Borson S, Scanlan JM, Watanabe J, Tu SP, Lessig M. Simplifying detection of cognitive impairment: comparison of the Mini-cog and 
Mini-mental state examination in a multiethnic sample. J Am Geriatr Soc. 2005;53(5):871-4.

54. Larner A. Short Montreal cognitive assessment: validation and reproducibility. J Geriatr Psychiatry Neurol. 2017;30(2):104-8.

55. Cole MG, Dastoor DP. A new hierarchic approach to the measurement of dementia. Accurate results within 15 to 30 minutes. Psychosomatics. 1987;28(6):298-301.

56. Mahoney Fl. Functional evaluation: the Barthel index. Maryland State Med J. 1965;14:61-5.

57. Charlson ME, Pompei P, Ales KL, Mackenzie CR. A new method of classifying prognostic comorbidity in longitudinal studies: development and validation. J Chronic Dis. 1987;40(5):373-83.

58. Rozzini R, Frisoni GB, Ferrucci L, Barbisoni P, Sabatini T, Ranieri P, et al. Geriatric index of comorbidity: validation and comparison with other measures of comorbidity. Age Ageing. 2002;31(4):277-85.

59. Rockwood K, Mitnitski A. How might deficit accumulation give rise to frailty. J Frailty Aging. 2012;1 (1):7-10.

60. Carnahan RM, Lund BC, Perry PJ, Pollock BG, Culp KR. The anticholinergic drug scale as a measure of drug-related anticholinergic burden: associations with serum anticholinergic activity. J Clin Pharmacol. 2006;46(12):1481-6.

61. Campbell MK, Piaggio G, Elbourne DR, Altman DG, Group C. Consort 2010 statement: extension to cluster randomised trials. BMJ (Clinical research ed). 2012;345:e5661.
62. Boutron I, Moher D, Altman DG, Schulz KF, Ravaud P, Group C. Extending the CONSORT statement to randomized trials of nonpharmacologic treatment: explanation and elaboration. Ann Intern Med. 2008;148(4):295-309.

63. Cole SR, MA. H. Constructing inverse probability weights for marginal structural models. Am J Epidemiol. 2008;168:656-64.

64. Fitzmaurice G. Methods for handling dropouts in longitudinal clinical trials. Statistica Neerlandica. 2003;57:75-99.

65. Little R, Rubin D. Statistical analysis with missing data. 2nd ed. New York: Wiley; 2001.

66. Rubin D. In: Son JW, editor. Multiple Imputation for Nonresponse in Surveys. New York: Wiley; 1987.

67. Leslie DL, Zhang Y, Bogardus ST, Holford TR, Leo-Summers LS, Inouye SK. Consequences of preventing delirium in hospitalized older adults on nursing home costs. J Am Geriatr Soc. 2005;53(3):405-9.

\section{Publisher's Note}

Springer Nature remains neutral with regard to jurisdictional claims in published maps and institutional affiliations.
Ready to submit your research? Choose BMC and benefit from:

- fast, convenient online submission

- thorough peer review by experienced researchers in your field

- rapid publication on acceptance

- support for research data, including large and complex data types

- gold Open Access which fosters wider collaboration and increased citations

- maximum visibility for your research: over 100M website views per year

At BMC, research is always in progress.

Learn more biomedcentral.com/submissions 